\title{
Giant topological and planar Hall effect in $\mathrm{Cr}_{1 / 3} \mathrm{NbS}_{2}$
}

\author{
D. A. Mayoh $\odot,{ }^{*}$ J. Bouaziz, A. E. Hall $\odot$, J. B. Staunton $\odot$, M. R. Lees $\odot$, and G. Balakrishnan $\odot^{\dagger}$ \\ Physics Department, University of Warwick, Coventry, CV4 7AL, United Kingdom
}

(Received 10 May 2021; revised 29 September 2021; accepted 30 November 2021; published 18 February 2022)

\begin{abstract}
$\mathrm{Cr}_{1 / 3} \mathrm{NbS}_{2}$ is a transition metal dichalcogenide that has been of significant interest due to its ability to host a magnetic chiral soliton lattice. Conventional and planar Hall measurements provide valuable insight into the detection of exotic spin structures in chiral magnets. We show that the presence of a giant planar Hall effect (PHE) can be attributed to a tilted soliton lattice in $\mathrm{Cr}_{1 / 3} \mathrm{NbS}_{2}$. Our detailed angular-dependent study shows the PHE and anisotropic magnetoresistance are intrinsically linked in complex noncoplanar magnets. From the conventional Hall signal we show the presence of a giant unconventional, likely topological Hall component that is the fingerprint of noncoplanar spin textures.
\end{abstract}

DOI: 10.1103/PhysRevResearch.4.013134

\section{INTRODUCTION}

The fundamental characteristics of two-dimensional (2D) magnetic materials can be scrutinized by examining the behavior of their electrons in magnetic and electric fields. Two-dimensional magnetic materials have been found to be highly tunable in terms of their physical characteristics making them excellent candidates for the study of exotic quantum phases and for spintronic devices $[1,2]$. Chiral helimagnetism (CHM) has provided an exciting twist in the story of $2 \mathrm{D}$ and bulk magnetic materials with various examples of selflocalized spin textures having been reported experimentally including skyrmions [3-5], anti-skyrmions [6], bubbles [7], and vortices [8]. These magnetic states often emerge due to the Dzyaloshinskii-Moriya interaction (DMI) $[9,10]$ but other stabilization mechanisms can also be at play, such as multispin interactions [11-13] or geometric frustration [14,15].

Hall measurements have proved to be an excellent tool for probing spin textures [16-19]. Indeed, the hallmark of skyrmion-like materials is the so-called topological Hall effect (THE) [20-22]. However, other forms of noncoplanar spin textures may also result in a finite THE. In particular, noncoplanar phases occurring in metallic compounds can cause variations in the magnetization which may lead to an emergent magnetic field that can deflect the electrons in the transverse direction [23]. A giant topological Hall coefficient is normally defined as $\rho_{\mathrm{xy}}^{\mathrm{THE}} \geqslant 1 \mu \Omega \mathrm{cm}$ and has been reported in several compounds including $\mathrm{Gd}_{2} \mathrm{PdSi}_{3}$ [22], $\mathrm{NdMn}_{2} \mathrm{Ge}_{2}$ [24], and thin film oxides [25].

Cr-intercalated $\mathrm{NbS}_{2}\left(\mathrm{Cr}_{1 / 3} \mathrm{NbS}_{2}\right)$ has attracted considerable attention due to the presence of a CHM state and, in an

\footnotetext{
*d.mayoh.1@warwick.ac.uk

†g.balakrishnan@warwick.ac.uk

Published by the American Physical Society under the terms of the Creative Commons Attribution 4.0 International license. Further distribution of this work must maintain attribution to the author(s) and the published article's title, journal citation, and DOI.
}

applied field, the observation of a chiral soliton lattice (CSL) by Lorentz transmission electron microscopy (LTEM) [26]. $\mathrm{Cr}_{1 / 3} \mathrm{NbS}_{2}$ is a member of the intercalated transition metal dichalcogenide family and has a chiral noncentrosymmetric hexagonal structure (space group: $P 6_{3} 22$ ) $[27,28]$. $\mathrm{Cr}_{1 / 3} \mathrm{NbS}_{2}$ consists of layers of $\mathrm{Nb}$ and $\mathrm{S}$ with $\mathrm{Cr}$ intercalated between the layers as shown in Fig. 1(a). It is known to have a complex magnetic phase diagram and five distinct magnetic phases have been detected through a variety of experimental techniques including LTEM, muon spin rotation/relaxation, magnetization, magnetocaloric effects, and magnetotransport [29]. The magnetic ordering observed in $\mathrm{Cr}_{1 / 3} \mathrm{NbS}_{2}$ is highly dependent on the experimental conditions. In zeroapplied field, a twisting array of magnetic moments in the $a b$ plane of $\mathrm{Cr}_{1 / 3} \mathrm{NbS}_{2}$ results in CHM ordering [26,30]. Applying a magnetic field along the screw axis (the $c$ axis) results in the magnet moments being tilted toward the direction of the field giving a chiral conical (CC) phase [31], while increasing the magnetic field still further results in a forced ferromagnetism (FFM). If the magnetic field is applied perpendicular to the screw axis, a CSL is established. This lattice is formed of areas of FFM broken by a twist in the magnetic field [26]. Recently, torque magnetometry has revealed a tilted CSL phase in $\mathrm{Cr}_{1 / 3} \mathrm{NbS}_{2}$ when the magnetic field is applied at an angle greater than 1.5 degrees from the $c$ axis [31].

The key finding of this work is the presence of a giant THE in $\mathrm{Cr}_{1 / 3} \mathrm{NbS}_{2}$ that is the fingerprint of noncoplanar spin textures in this material. The emergence of a finite planar Hall effect (PHE) is attributed to the presence of the tilted CSL. The temperature and field dependence of the PHE has been thoroughly investigated in parallel with its longitudinal counterpart, the anisotropic magnetoresistance (AMR). Both are found to be intimately linked, highlighting that the connection between the PHE and the AMR is still valid for complex noncoplanar magnets. Furthermore, by disentangling the ordinary and anomalous Hall components from the conventional Hall data, we reveal the presence of a giant THE. We attribute the origin of the THE in $\mathrm{Cr}_{1 / 3} \mathrm{NbS}_{2}$ to the complex magnetic textures present in this material. 

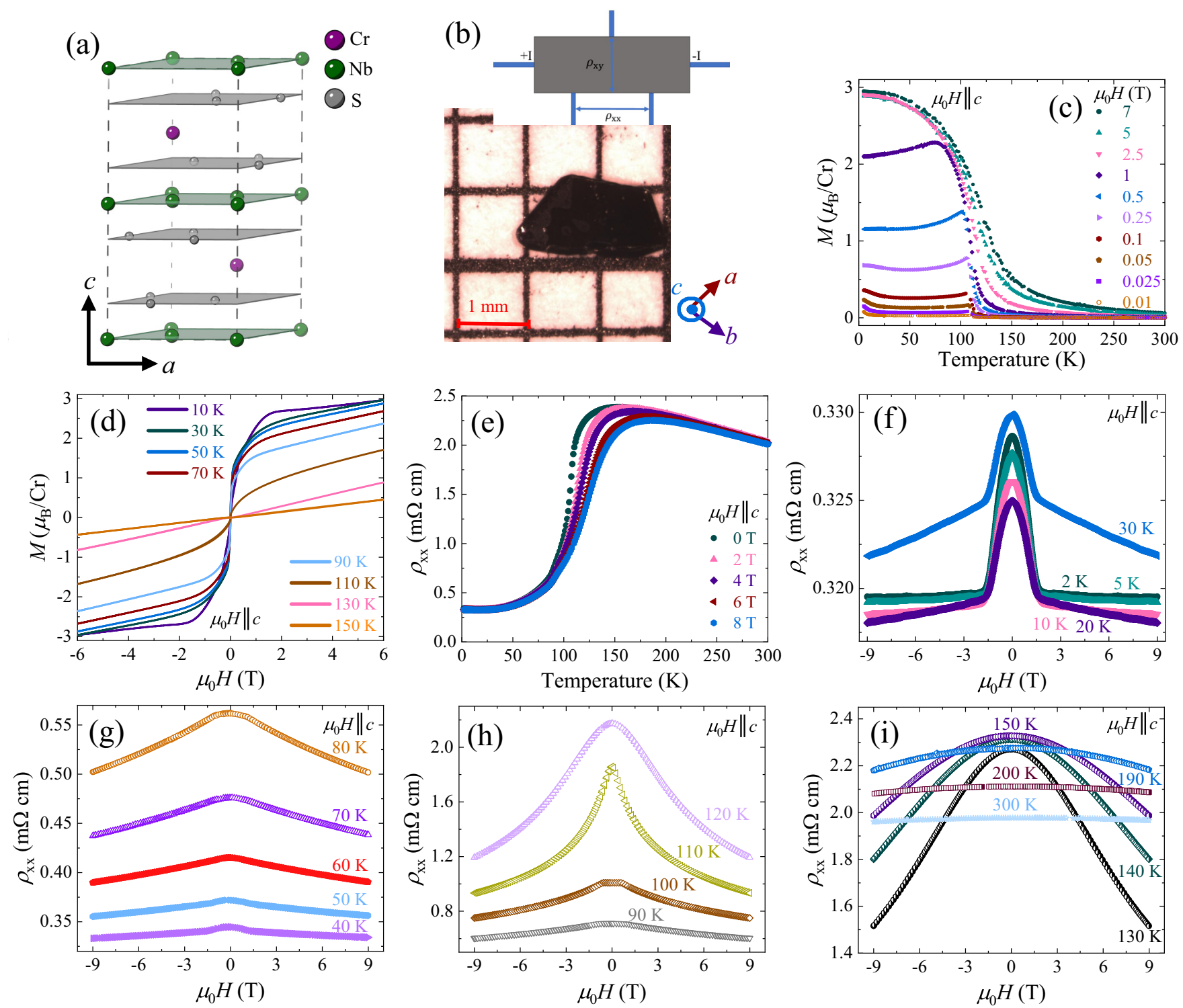

FIG. 1. (a) Crystal structure of $\mathrm{Cr}_{1 / 3} \mathrm{NbS}_{2}$. The purple, green, and gray spheres denote the chromium, niobium, and sulfur atoms, respectively. The dashed line indicates the unit cell. (b) A single crystal of $\mathrm{Cr}_{1 / 3} \mathrm{NbS}_{2}$ grown by chemical vapor transport that was used for all the measurements presented in this work, along with a schematic of the 5-point wire configuration used for the longitudinal and Hall resistivity measurements. (c) Magnetization versus temperature curves for $\mathrm{Cr}_{1 / 3} \mathrm{NbS}_{2}$ for fields of up to $7 \mathrm{~T}$ applied perpendicular to the platelet. (d) Magnetization as a function of field applied parallel to the $c$ axis at several temperatures. (e) Temperature dependence of the longitudinal resistivity $\rho_{\mathrm{xx}}$ in several magnetic fields applied parallel to the $c$ axis. Magnetoresistances of $\mathrm{Cr}_{1 / 3} \mathrm{NbS}_{2}$ between -9 and $9 \mathrm{~T}$ at (f) 2 to $30 \mathrm{~K}$, (g) 40 to $80 \mathrm{~K}$, (h) 90 to $120 \mathrm{~K}$, and (i) 130 to $300 \mathrm{~K}$.

\section{EXPERIMENTAL DETAILS}

Single crystals of $\mathrm{Cr}_{1 / 3} \mathrm{NbS}_{2}$ were produced by the chemical vapor transport technique using iodine as the transport agent. Polycrystalline powders of $\mathrm{Cr}_{1 / 3} \mathrm{NbS}_{2}$ along with 5 $\mathrm{mg} / \mathrm{cc}$ of the transport agent were placed in an evacuated and sealed quartz tube. The tube was then heated with one end held at $950{ }^{\circ} \mathrm{C}$ and the other at $800{ }^{\circ} \mathrm{C}$ for 3 weeks before cooling to room temperature. Several single crystals in the form of hexagonal platelets with sides $\sim 2 \mathrm{~mm}$ in length were obtained in each growth. DC magnetization, $M$, measurements were performed as a function of temperature
$(1.8$ to $300 \mathrm{~K})$ and field $(0$ to $7 \mathrm{~T})$ in a Quantum Design Magnetic Property Measurement System. Magnetic hysteresis loops were acquired using an Oxford Instruments vibrating sample magnetometer in magnetic fields up to $6 \mathrm{~T}$. Resistivity, conventional Hall, and PHE measurements were performed as a function of temperature $(1.8$ to $300 \mathrm{~K})$ and field $(0$ to $9 \mathrm{~T}$ ) using the ac transport option in a Quantum Design Physical Property Measurement System. Currents between 1 and $10 \mathrm{~mA}$ at a frequency of $113 \mathrm{~Hz}$ were used for all the measurements. Further details on the fitting procedure used for the conventional Hall effect data can be found in Appendix A. 


\section{MAGNETIZATION AND LONGITUDINAI RESISTIVITY}

A single crystal of $\mathrm{Cr}_{1 / 3} \mathrm{NbS}_{2}$ with a $T_{\mathrm{c}}$ of $111 \mathrm{~K}$ was chosen for these experiments as shown in Fig. 1(b). A Laue diffraction pattern of the crystal is shown in the Supplemental Material [32]. DC magnetization, $M$, versus temperature curves for the single crystal of $\mathrm{Cr}_{1 / 3} \mathrm{NbS}_{2}$ in various fields up to $7 \mathrm{~T}$ applied perpendicular to the platelet are shown in Fig. 1(c). In low fields the system exhibits a sharp transition to a magnetically ordered state at $T_{\mathrm{c}}=111 \mathrm{~K}$. As the field is increased the magnetic transition broadens. In $7 \mathrm{~T}$ we observe a significant magnetization up to $250 \mathrm{~K}$. Magnetization as a function of applied field, $H$, where the field was applied nominally along the $c$ axis is shown in Fig. 1(d). At low temperature the magnetization increases with increasing applied field until saturation at $M=2.9(1) \mu_{\mathrm{B}} / \mathrm{Cr}$ atom. The saturation magnetization of $\mathrm{Cr}_{1 / 3} \mathrm{NbS}_{2}$ is expected to be approximately $3 \mu_{\mathrm{B}} / \mathrm{Cr}$ atom for chromium in the trivalent $(\mathrm{S}=3 / 2)$ state. The magnetization curves can be explained by the presence of the CHM slowly being polarized along the direction of the applied field. No significant hysteresis is visible in the $M(H)$ curves at any temperature.

The different magnetic regimes in $\mathrm{Cr}_{1 / 3} \mathrm{NbS}_{2}$ are observed to have distinct electronic transport properties. The temperature dependence of the zero-field longitudinal resistivity $\rho_{\mathrm{xx}}$ is shown in Fig. 1(e). Also shown in Figs. 1(f)-1(i) is $\rho_{\mathrm{xx}}$ versus temperature for assorted magnetic fields applied parallel to the $c$ axis. The temperature dependence of the longitudinal resistivity differs from a conventional metallic ferromagnet [33]; $\rho_{\mathrm{xx}}$ initially increases with decreasing temperature until $\sim 150 \mathrm{~K}$. A similar increase in $\rho_{\mathrm{xx}}$ is observed in $\mathrm{UPd}_{2} \mathrm{Si}_{2}$ and was attributed to the Kondo effect [34]. Below $150 \mathrm{~K}$ the resistivity of $\mathrm{Cr}_{1 / 3} \mathrm{NbS}_{2}$ then falls over a wide temperature interval, $\Delta T=100 \mathrm{~K}$, centered around $T_{\mathrm{c}}$. The drop in the resistivity at temperatures below the magnetic transition is due to the magnetic ordering which diminishes the spin-disorder scattering [35]. This reduction is not monotonous but instead tends to saturate as observed in previous work [36]. A possible origin of this behavior is a disorder of the $\mathrm{Cr}$ atoms [37]. However, given the presence of a helical phase/CSL in this temperature regime, there is no one-to-one correspondence between the behavior seen here and that observed in a simple ferromagnet. The residual resistivity at $2 \mathrm{~K}$ is $\rho_{\mathrm{xx}, 0}=$ $0.339(5) \mathrm{m} \Omega \mathrm{cm}$.

The field dependence of $\rho_{\mathrm{xx}}$ in $\mathrm{Cr}_{1 / 3} \mathrm{NbS}_{2}$ for a selection of temperatures is shown in Figs. 1(e)-1(h). Different behaviors are observed in the various temperature regimes dependent on the magnetic ordering. Between 2 and $20 \mathrm{~K} \rho_{\mathrm{xx}}$ is seen to saturate in fields above $2 \mathrm{~T}$. The rapid decrease in the resistivity from 0 to $2 \mathrm{~T}$ can be attributed to the presence of the CSL, and as the field is increased the moments are canted toward the direction of field which in turn diminishes the spin-disorder scattering; $\rho_{\mathrm{xx}}$ saturates when the spins are fully polarized along $H$. This field dependence of the magnetoresistance is typical of helimagnetic systems [38,39]. A plateau in the magnetoresistance has also been attributed to a skyrmion lattice phase in $\mathrm{Gd}_{2} \mathrm{PdSi}_{3}$ [40]. Between 20 and $110 \mathrm{~K}$, the distinct change in slope of $\rho_{\mathrm{xx}}(H)$ around $2 \mathrm{~T}$ becomes more diffuse as the mean-free path of the electrons

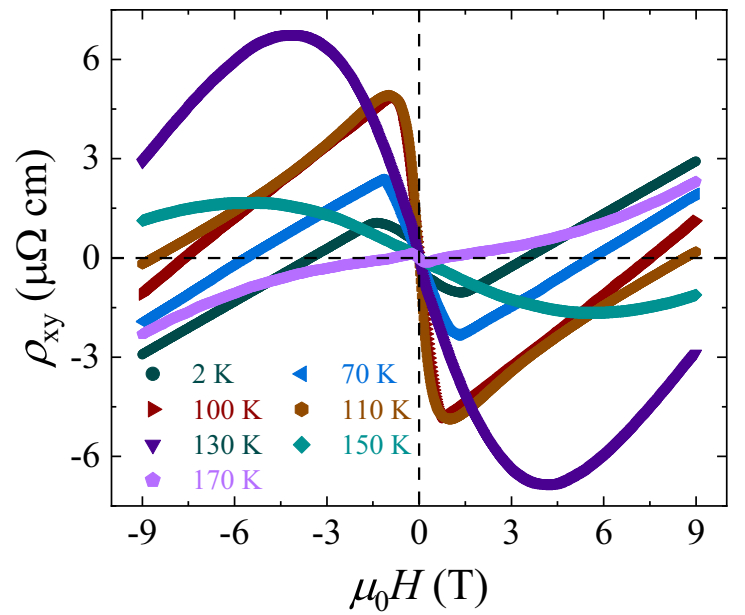

FIG. 2. Field-dependent Hall resistivity $\rho_{\mathrm{xy}}$ at 10, 70, 110, 130, 190 , and $230 \mathrm{~K}$. There is a significant change in the behavior of the Hall effect as the temperature is lowered through $T_{\mathrm{c}}$. It is observed that the shape of the field dependence in Hall effect changes from a smooth almost parabolic shape to a sharp triangular shape.

decreases; therefore they experience less of the CSL phase. At temperatures above $120 \mathrm{~K}$, which is above $T_{\mathrm{c}}, \rho_{\mathrm{xx}}$ is seen to have parabolic dependence, typical of ferromagnetic materials, before becoming almost linear with $H$ at $300 \mathrm{~K}$.

\section{HALL EFFECT IN $\mathrm{Cr}_{1 / 3} \mathrm{NbS}_{2}$}

Hall effect measurements on $\mathrm{Cr}_{1 / 3} \mathrm{NbS}_{2}$ were performed at a variety of temperatures, where the field was applied perpendicular to the largest face of the crystal platelet (nominally along the $c$ axis) and the current applied within the plane of the plate (nominally the $a b$ plane), and are shown in Fig. 2. To understand the underlying physics in $\mathrm{Cr}_{1 / 3} \mathrm{NbS}_{2}$ we investigate the Hall response and disentangle its different components. The Hall signal is usually made up of several contributions:

$$
\rho_{x y}=\rho_{x y}^{\mathrm{OHE}}+\rho_{x y}^{\mathrm{AHE}}+\rho_{x y}^{\mathrm{THE}} .
$$

The first term $\rho_{\mathrm{xy}}^{\mathrm{OHE}}$ is the ordinary Hall effect (OHE) which scales linearly with the magnetic field $B, \rho_{\mathrm{xy}}^{\mathrm{OHE}}=R_{\mathrm{H}} B$, where $R_{\mathrm{H}}$ is the Hall coefficient. The second term $\rho_{\mathrm{xy}}^{\mathrm{AHE}}$ is the anomalous Hall effect (AHE), which emerges from two mechanisms, one intrinsic and the other extrinsic. The intrinsic contribution is related to the presence of a nonvanishing Berry curvature leading to an internal magnetic field which deflects the electrons in a direction perpendicular to the applied electric field $[42,43]$. The extrinsic contribution can be related to side jump and skew scattering [43-45]. Usually the skew scattering contribution is the weakest component; however, it has been shown recently that it can have a measurable impact on the Hall response in some materials [46]. The third term, $\rho_{\mathrm{xy}}^{\mathrm{THE}}$, is the THE $[18,20,47]$ whose dependence on the magnetization is nontrivial, $\rho_{\mathrm{xy}}^{\mathrm{THE}}$ is proportional to the emergent field $\vec{B}_{\mathrm{e}}$ created by a noncoplanar magnetic texture [23,48], where $\vec{B}_{\mathrm{e}} \propto \vec{m} \cdot\left(\frac{\partial \vec{m}}{\partial x} \times \frac{\partial \vec{m}}{\partial y}\right) ; \rho_{\mathrm{xy}}^{\mathrm{THE}}$ has a nonlinear evolution with the magnetic field and is only seen in the presence of noncoplanar phases [23]. This component of the Hall signal is antisymmetric when the direction of the applied magnetic field is 
reversed [25]. At this point it worth considering whether any of the magnetic phases in $\mathrm{Cr}_{1 / 3} \mathrm{NbS}_{2}$ can generate a topological Hall contribution. Neither the helimagnetic phase nor the CSL will display a THE since the scalar chirality must vanish, i.e., $\mathcal{C}_{i j k}=\vec{m}_{i} \cdot\left(\vec{m}_{j} \times \vec{m}_{k}\right)$. However, for the case of a tilted-CSL [31], a finite THE contribution is possible (see Appendix C).

The presence of tilted CSL may also lead to the appearance of a PHE in $\mathrm{Cr}_{1 / 3} \mathrm{NbS}_{2}$. The PHE emerges solely from the AMR [49-51], and occurs when the in-plane component of the magnetization is misaligned with respect to the applied current. For a current along the $x$ direction, $\rho_{x y}^{\mathrm{PHE}}$ is [50-52]:

$$
\rho_{x y}^{\mathrm{PHE}}=\left(\rho_{\|}-\rho_{\perp}\right) m_{x} m_{y},
$$

where $m_{x}$ and $m_{y}$ are the in-plane components of the magnetization and $\rho_{\|}\left(\rho_{\perp}\right)$ are the longitudinal resistivity when $\vec{m}$ is parallel (perpendicular) to the current direction.

\section{PLANAR HALL EFFECT AND ANISOTROPIC MAGNETORESISTANCE}

One of the keys to understanding the magnetic spin textures in our Hall effect measurements is the presence of a PHE. Indeed, a PHE and its longitudinal counterpart, an AMR, are observed in $\mathrm{Cr}_{1 / 3} \mathrm{NbS}_{2}$. The presence of the PHE indicates a finite in-plane magnetization in the magnetic structure. Thus we consider the two possible scenarios currently proposed for $\mathrm{Cr}_{1 / 3} \mathrm{NbS}_{2}$ when the field is applied close to the $c$ axis: A $\mathrm{CC}$ phase (when the offset is less than $1.5^{\circ}$ ) and a tilted CSL (when the offset is greater than $1.5^{\circ}$ ) [31]. In the $\mathrm{CC}$ phase the magnetic moments provide a net magnetic moment that points directly along the helical axis (out of plane) due to symmetry. Once the field is sufficient to polarize all the moments along the helical axis, the magnetic moments continue to be out of plane. Hence, the absence of any in-plane magnetization from the CC phase and eventual FFM phase means no PHE would be observed. In the case of a tilted CSL where all the magnetic moments are uniformly canted toward the helical axis, it is easy to see that there is a net magnetic moment that is neither perpendicular nor parallel to the helical axis. This then means that an in-plane magnetization vector does exist; however, it is tilted slightly out of plane. It is important to note that the PHE can persist outside of the tilted CSL phase as the slightly out-of-plane magnetization persists once FFM is stabilized due to any misalignment of the field and the plane. The different magnetic configurations occurring when the external field is applied in different directions are depicted in Fig. 3(a). For our conventional Hall effect measurements we apply the field perpendicular to the plane of the platelet, which is assumed to be nominally close to $c$ axis. Taking this symmetry arrangement of current and magnetic field along with the modeled spin structures of the magnetic textures we conclude that the PHE arises from the magnetic moments in the tilted CSL and that they are pointing between the $c$ axis and the $a b$ plane.

For $\mathrm{Cr}_{1 / 3} \mathrm{NbS}_{2}$ the CSL has been observed via LTEM when imaging along the $a b$ plane with a field applied perpendicular to the $c$ axis [53]. For our magnetotransport measurements the current is applied along the length of the platelet, which is nominally in the $a b$ plane. For the conventional Hall effect measurements we apply the field perpendicular to the plane of the platelet, which is assumed to be nominally along the $c$ axis. Taking this symmetry arrangement of current and magnetic field along with the modeled spin structures of the magnetic textures described in the previous section we conclude that the magnetic moments in the tilted CSL are pointing between the $c$ axis and the $a b$ plane.

The PHE and AMR as a function of angle in $\mathrm{Cr}_{1 / 3} \mathrm{NbS}_{2}$ are shown in Figs. 3(b) and 3(c). The angle $\varphi$ between the current and the applied field is defined in Fig. 3(b). A clear oscillatory signal of the form $\sin \varphi \cos \varphi$ for the PHE and $\sin ^{2} \varphi$ for the AMR is observed and can be fit by Eq. (2). The amplitude of the oscillations gives the magnitudes of the PHE and AMR.

The observed temperature and field dependence of the PHE and AMR supports the theory of a tilted-CSL in $\mathrm{Cr}_{1 / 3} \mathrm{NbS}_{2}$. It can be seen in Figs. 3(d) and 3(e) that in all fields, close to the magnetic transition, the magnitudes of both the PHE and the AMR amplitudes increase. The field dependence of the PHE above and below the magnetic transition are of particular note, as shown in Figs. 3(g) and 3(h), as there appear to be two components. First, there exists a low-field component below the magnetic transition where there is a rapid increase in the PHE amplitude below $100 \mathrm{mT}$. This component disappears above the transition temperature. We can therefore attribute this to the magnetic state as the transition from the CSL phase to the FFM phase is expected to occur around $100 \mathrm{mT}$ [54]. The high-field component is the traditional component we would expect to observe from a ferromagnetic system. Indeed, the maximum in the PHE amplitude at fields above $1 \mathrm{~T}$ approximately corresponds to the Curie temperature determined from magnetization. To obtain this PHE component the only requirement is that the sample has some finite in-plane magnetization which we have confirmed in our magnetization measurements [see Fig. 1(c)]. Further planar Hall resistivity as a function of field data across all measured temperatures is shown in the Supplemental Material [32].

A similar oscillatory signal, this time of the form $\sin ^{2} \varphi$, is also observed when measuring the longitudinal resistivity in this configuration as shown in Fig. 3(d). This longitudinal resistivity is generally referred to as the AMR. From the resistivity tensor it is expected that the amplitude of the PHE and AMR oscillations [i.e., $\left.\left(\rho_{\|}-\rho_{\perp}\right)\right]$ should be of equal magnitude [51]. However, any small misalignment of the sample can cause the ordinary magnetoresistance to leak into the AMR signal. The value for the PHE signal is significantly more reliable, however, as the effects of any leaking of the ordinary Hall effect into the PHE due to a misalignment is antisymmetric and can be removed by averaging the positive and negative field data.

\section{EMERGENCE OF A GIANT TOPOLOGICAL HALL EFFECT}

An antisymmetrization procedure was first performed on the Hall effect data to remove any symmetric components originating from the magnetoresistance and PHE to simplify the extraction of the THE component. The individual contributions to the Hall signal in $\mathrm{Cr}_{1 / 3} \mathrm{NbS}_{2}$, namely, the $\mathrm{OHE}$ and AHE are extracted from the remaining Hall signal following the procedure described in Appendix A. A finite and significant component to the Hall signal remains once the 
(a)

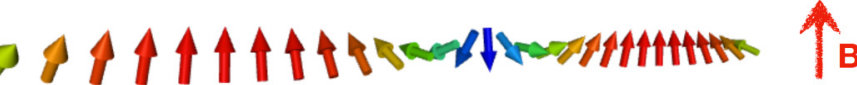
c-axis

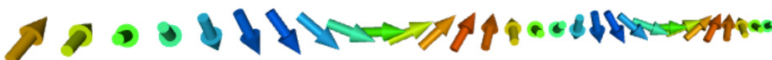

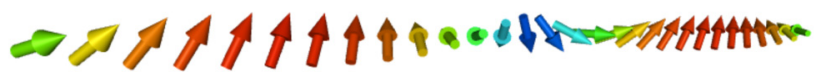

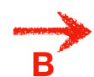

Temperature $(\mathrm{K})$
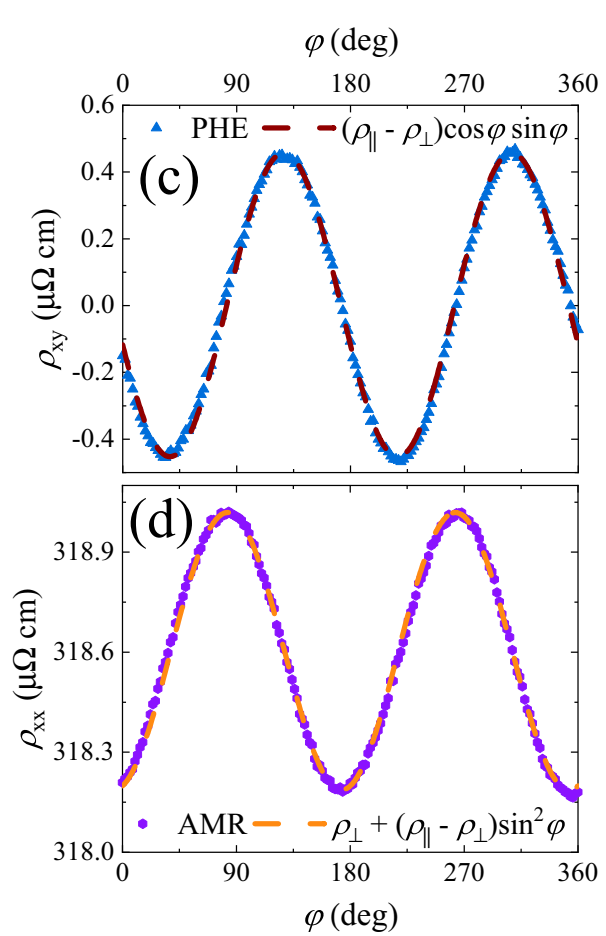

(b)

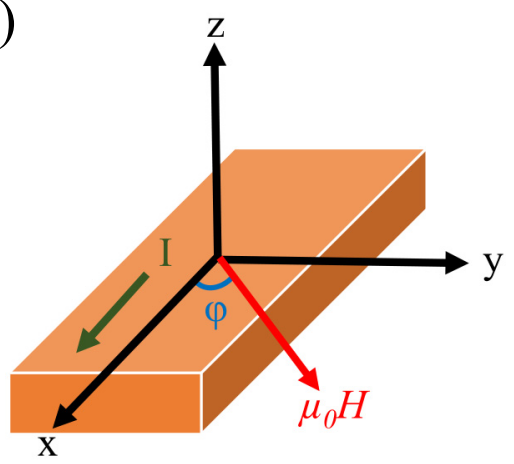

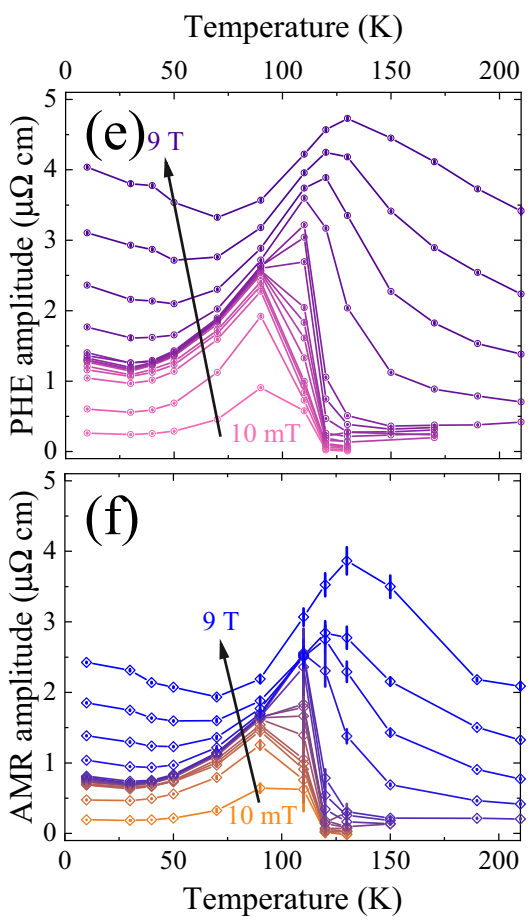

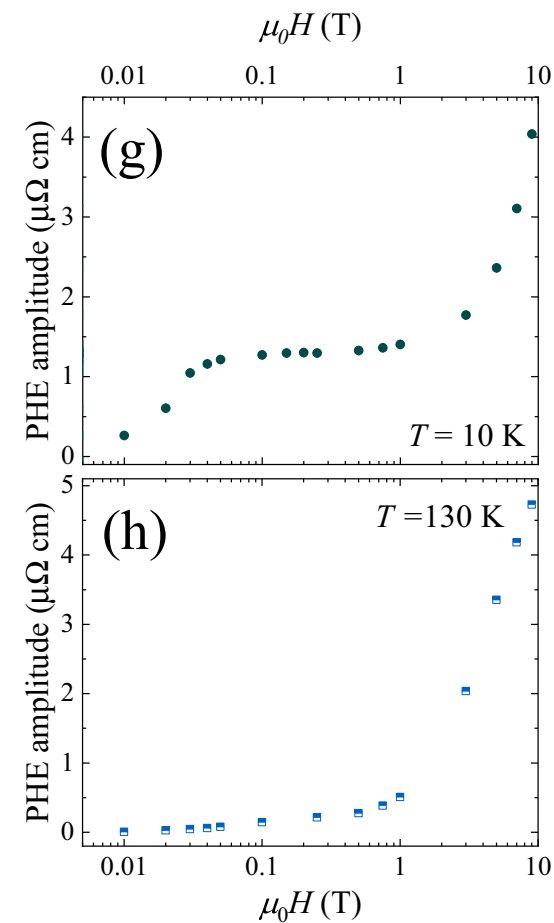

FIG. 3. (a) Possible magnetic ground states as a function of the magnetic field direction: CSL, CC phase, and tilted CSL. The chain contains 30 atoms, the model parameters are $J=10 \mathrm{meV}$ (exchange), $D=0.5 J$ (DMI), $B=6 \mathrm{~T}$ with a $45^{\circ}$ tilt with respect to the $c$ axis. The simulations were performed using the atomistic spin SPIRIT code [41]. Further details can be found in the Appendix B. (b) Schematic of the PHE and AMR set up. (c, d) PHE $\rho_{\mathrm{xy}}$ and AMR $\rho_{\mathrm{xx}}$ taken at $5 \mathrm{~K}$. The dashed lines indicate fits to the PHE and AMR (as described in the text). (e, f) PHE and AMR amplitudes, $\left(\rho_{\|}-\rho_{\perp}\right)$, as a function of temperature at various applied fields. The field dependence of the PHE amplitudes at (g) 10 and (h) $130 \mathrm{~K}$.

$\mathrm{OHE}$ and AHE are extracted; we attribute this contribution to the THE. The individual Hall components at several temperatures are shown in Figs. 4(a)-4(c) (further conventional Hall resistivity as a function of field data across all measured temperatures are shown in the Supplemental Material [32]). The ordinary Hall coefficient $R_{\mathrm{H}}$ is positive across all temperatures indicating hole-like conduction in $\mathrm{Cr}_{1 / 3} \mathrm{NbS}_{2}$; at $30 \mathrm{~K}, R_{\mathrm{H}}=7.31(2) \times 10^{-9} \mathrm{~m}^{3} / \mathrm{C}$ gives a hole density of $8.53(2) \times 10^{26} \mathrm{~m}^{-3}$ consistent with what has previously been reported [55]. The AHE component is seen to be always negative, diminishing in magnitude with increasing temperature. However, an anomalous Hall component is still noticeable well into the paramagnetic regime. This behavior has previously been observed in $\mathrm{MgZnO} / \mathrm{ZnO}$ heterostructures and explained using the Giovannini-Kondo model where localized paramagnetic centers can generate an anomalous Hall component from the skew scattering of electrons off these centers [56]. The skew, side, and intrinsic scattering terms are all found to be significant below the magnetic transition temperature, gradually increasing in magnitude as the temperature decreases. The scattering term can be a useful measure of the quality of crystal used as it is normally only expected to be significant in near perfect crystals $[43,57]$; hence the high quality of the crystals used in this experiment is particularly notable.

Finally we discuss $\rho_{\mathrm{xy}}^{\mathrm{THE}}$ in the light of CSL for $\mathrm{Cr}_{1 / 3} \mathrm{NbS}_{2}$. The temperature dependence of the maximum magnitude of $\rho_{\mathrm{xy}}^{\mathrm{THE}}$ is shown in Fig. 4(d). At the transition temperature the 

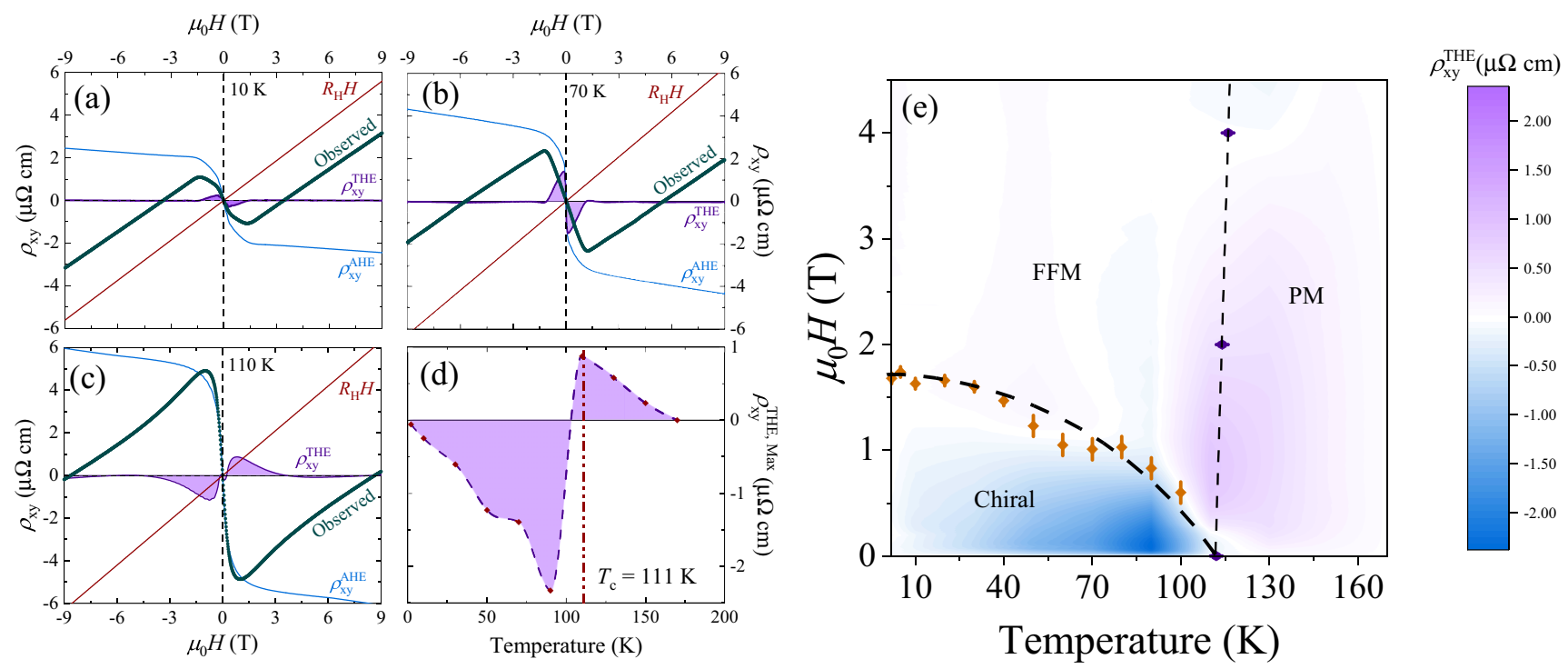

FIG. 4. Observed $\rho_{x y}$ (green) as a function of applied magnetic field at (a) 10, (b) 70, and (c) $110 \mathrm{~K}$ with the calculated contributions of $\rho_{\mathrm{xy}}^{\mathrm{OHE}}$ (red), $\rho_{\mathrm{xy}}^{\mathrm{AHE}}$ (blue), and $\rho_{\mathrm{xy}}^{\mathrm{THE}}$ (purple). (d) The maximum $\rho_{\mathrm{xy}}^{\mathrm{THE}}$ as a function of temperature. (e) Contour map of $\rho_{\mathrm{xy}}^{\mathrm{THE}}$ as function of field and temperature. The purple points indicate the position of the transition from resistivity as seen in Fig. 1(b). The phase boundaries are approximated from the $\rho_{\mathrm{xx}}$ versus temperature (purple) and field (orange) data shown in Fig. 1. The dashed lines are estimations of phase boundaries in $\mathrm{Cr}_{1 / 3} \mathrm{NbS}_{2}$ marking out the chiral, FFM, and paramagnetic (PM) phases.

sign of the THE is seen to flip from positive $\left(T>T_{\mathrm{c}}\right)$ to negative $\left(T<T_{\mathrm{c}}\right)$. Below the magnetic transition the THE has a maximum magnitude of $2.33 \mu \Omega \mathrm{cm}$ at $90 \mathrm{~K}$. The change in the sign of the $\rho_{\mathrm{xy}}^{\mathrm{THE}}$ is likely indicative of a change in the spin polarization of the charge carriers as the temperature passes through the transition. Parallels can be drawn and discussed with the AHE and how it behaves at the phase boundary. It has been shown that due to thermal spin fluctuations, the skew scattering contributions to the AHE become more important and can cause a change of sign [58]. Assuming that the THE is similar to the AHE, with the main difference being that the THE emerges from noncoplanar spins instead of spinorbit coupling, one may conjecture that a similar mechanism occurs at the phase transition. $\rho_{\mathrm{xy}}^{\mathrm{THE}}$ is significantly larger than has been reported in other helical ground state antiferromagnets such as $\mathrm{MnSi}(\sim-7 \mathrm{n} \Omega \mathrm{cm})[20,59]$ and $\mathrm{FeGe}$ $(\sim-136 \mathrm{n} \Omega \mathrm{cm})$ [21]. Both $\mathrm{MnSi}$ and FeGe host skyrmion lattices which are thought to only induce a small THE. This suggests that the mechanism for the THE in $\mathrm{Cr}_{1 / 3} \mathrm{NbS}_{2}$ is provided through some other magnetic spin texture, namely, the tilted CSL. Similarly, if the rotation period is smaller, then the emergent field is expected to be larger, which can lead to a larger $\rho_{\mathrm{xy}}^{\mathrm{THE}}$. It is expected that the change of period will lead to a nonlinear change in the topological charge density once the tilted CSL is stabilized since $\mathcal{C}_{i j k}=\vec{m}_{i} \cdot\left(\vec{m}_{j} \times \vec{m}_{k}\right)$; hence important changes in the THE will occur. Furthermore, the THE is a complex phenomena and depends on other details of the electronic structure such as the scattering rate off the magnetic atoms and the density of the charge carriers. A contour map of $\rho_{\mathrm{xy}}^{\mathrm{THE}}$ [see Fig. 4(e)] reveals the presence of $\rho_{\mathrm{xy}}^{\mathrm{THE}}$ across most temperatures below the transition temperature before becoming negligible at $2 \mathrm{~K}$. As the temperature decreases, the fluctuations in the magnetic spins from the chiral phase would be expected to reduce. Therefore as the temperature is reduced the magnitude of the THE would be expected to reduce as well. The region of increased magnitude in $\rho_{\mathrm{xy}}^{\mathrm{THE}}$ corresponds to the same region where the CSL has been reported by LTEM $[26,30,60]$ and ac magnetization [61]. This strongly suggests that the $\rho_{\mathrm{xy}}^{\mathrm{THE}}$ is due to a noncoplanar spin structure arising from a tilted CSL.

\section{SUMMARY}

Remarkably, the chiral helimagnet $\mathrm{Cr}_{1 / 3} \mathrm{NbS}_{2}$ is found to host both a giant THE and PHE. We show that the observation of a PHE and THE in $\mathrm{Cr}_{1 / 3} \mathrm{NbS}_{2}$ indicates the presence of a tilted CSL. Furthermore, the close correspondence of the PHE and AMR in this complex noncoplanar magnet validates the theory that the connection between these two quantities still holds in these more complex systems. The conventional Hall measurements performed in this study demonstrate that noncoplanar spins arising from a tilted CSL can indeed generate the emergent field necessary for the THE. Studying $\mathrm{Cr}_{1 / 3} \mathrm{NbS}_{2}$, where the phase diagram has been extensively mapped out, has allowed a one-to-one correspondence to be drawn between the relative magnetic phases and the magnetotransport properties observed. Notably, the THE exhibited in $\mathrm{Cr}_{1 / 3} \mathrm{NbS}_{2}$ is several orders of magnitude larger than those of similar helimagnetic materials where skyrmions are observed. This study marks an important step forward in demonstrating that magnetotransport measurements, and more specifically the THE can be used to detect not only skyrmions but also noncoplanar spin textures such as a tilted CSL.

Data will be made available via Warwick Research Archive Portal [62]. 


\section{ACKNOWLEDGMENTS}

We acknowledge Tom Orton, Patrick Ruddy, and Daisy Ashworth for their technical support. This work was financially supported by three Engineering and Physical Sciences Research Council grants: EP/T005963/1, EP/M028941/1 (PRETAMAG), and the UK Skyrmion Project Grant $\mathrm{EP} / \mathrm{N} 032128 / 1$.

\section{APPENDIX A: FITTING PROCEDURE FOR THE HALL EFFECT}

To estimate the contribution the THE makes to the Hall resistivity, the following fitting procedure was used. To simplify the problem we first remove any Hall contributions from the PHE and any magnetoresistance. To do this an antisymmetrization procedure was performed using $\rho(+H)^{\prime}=\left[\rho\left(+H^{\mathrm{inc}}\right)-\rho\left(-H^{\mathrm{dec}}\right)\right] / 2$ and $\rho(-H)^{\prime}=$ $\left[\rho\left(-H^{\text {inc }}\right)-\rho\left(+H^{\text {dec }}\right)\right] / 2$ where $\rho\left(+H^{\text {inc }}\right)$ is the Hall resistivity in the positive field quadrants when the field is increasing in magnitude, $\rho\left(+H^{\mathrm{dec}}\right)$ is the Hall resistivity in the positive field quadrants when the field is decreasing in magnitude, $\rho\left(-H^{\text {inc }}\right)$ is the Hall resistivity in the negative field quadrants when the field is increasing in magnitude and $\rho\left(-H^{\text {inc }}\right)$ is the Hall resistivity in the negative field quadrants when the field is decreasing in magnitude. For fields greater than some critical field value, $\rho_{\mathrm{xy}}^{\mathrm{THE}}$ should be equal to zero as FFM should have no spin chirality. The contribution from the ordinary Hall effect $\rho_{\mathrm{xy}}^{\mathrm{OHE}}$ and the anomalous Hall effect $\rho_{\mathrm{xy}}^{\mathrm{AHE}}$ can be calculated by linearizing the data using $\rho_{\mathrm{xy}} / B=$ $\alpha \rho_{\mathrm{xx}}^{2} M / H+R_{\mathrm{H}}$ (not shown). Here $\alpha=\left(S_{\text {Int }}+S_{\text {Side }}\right)$, where $S_{\text {Int }}$ is the intrinsic contribution and $S_{\text {Side }}$ is the side jump contribution, only provides the $\rho_{\mathrm{xx}}^{2}$ contribution for the AHE but we now have a value for $R_{\mathrm{H}}$. To estimate the full contribution of $\rho_{\mathrm{xy}}^{\mathrm{AHE}}$ we can subtract $\rho_{\mathrm{xy}}^{\mathrm{OHE}}$ from the total Hall resistivity and by using the following equality $\left(\rho_{\mathrm{xy}}-\rho_{x y}^{\mathrm{OHE}}\right) / \rho_{\mathrm{xx}} M=$ $\alpha \rho_{\mathrm{xx}}+S_{\text {Skew }}$, where $S_{\text {Skew }}$ is the skew scattering contribution, the data can be linearized. This allows for $\alpha$ and $S_{\text {Skew }}$ to be extracted, (not shown).

\section{APPENDIX B: SIMULATIONS OF THE SPIN DYNAMICS}

The magnetic state of the system is described by an extended Heisenberg Hamiltonian:

$$
\begin{aligned}
\mathcal{E}_{M}= & -\frac{1}{2} \sum_{i j} J_{i j} \vec{M}_{i} \cdot \vec{M}_{j}+\frac{1}{2} \sum_{i j} \vec{D}_{i j} \cdot\left(\vec{M}_{i} \times \vec{M}_{j}\right) \\
& -\sum_{i} \vec{M}_{i} \cdot \vec{B} .
\end{aligned}
$$

The first term in the Hamiltonian designates the isotropic Heisenberg exchange interaction, the second is the DMI, and the third is the Zeeman coupling to an external magnetic field. The following parameters are used: A nearest-neighbor exchange $J_{i j}=10.0 \mathrm{meV}$, and nearest-neighbor DMI $D=$ $5.0 \mathrm{meV}$, and magnetic moment of $M=3 \mu_{\mathrm{B}}$ with magnetic field of $6 \mathrm{~T}$. The field is oriented in two directions in practice either along the $z$ or tilted at $45^{\circ}$ away from the $z$ axis in (a)

\section{Helimagnet (A)}

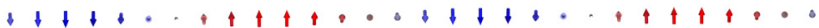

Chiral soliton lattice $(\mathbf{B})$

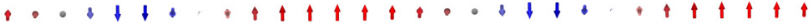

Tilted chiral soliton lattice $(\mathbf{C})$

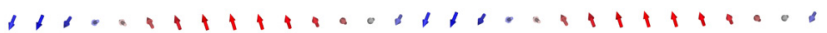

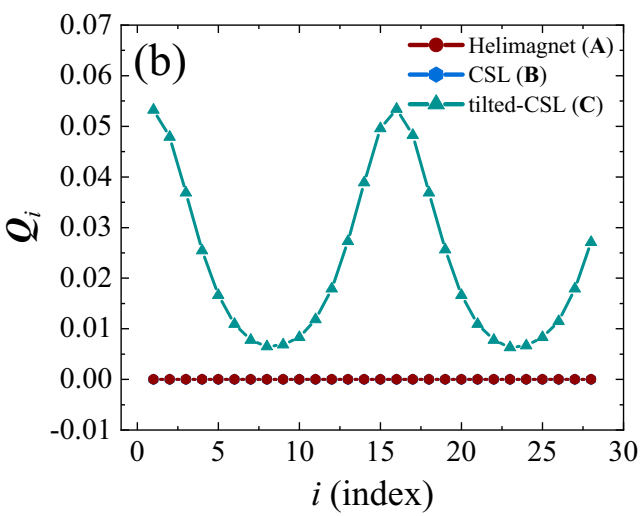

FIG. 5. (a) Three spin configurations possible in $\mathrm{Cr}_{1 / 3} \mathrm{NbS}_{2}$ : $\mathrm{He}-$ limagnet (A), CSL (B), and tilted CSL (C). (b) Evolution of the topological charge density for a Helimagnet (A), a CSL (B), and a tilted CSL (C). The topological charge density for the helimagnet state (A) is zero for all $i$ so is not visible.

the $x z$ plane. The minimization of the energy is performed using the SPIRIT code, which uses an algorithm based on the Landau-Lifshitz-Gilbert equation [41].

\section{APPENDIX C: TOPOLOGICAL HALL EFFECT FROM CHIRAL PHASES}

As discussed in the previous section, neither the helimagnetic phase nor the CSL will display a THE since the scalar chirality must vanish, i.e., $\mathcal{C}_{i j k}=\vec{m}_{i} \cdot\left(\vec{m}_{j} \times \vec{m}_{k}\right)$. However, for the case of a tilted CSL, a finite THE contribution is possible. To illustrate this we consider three different cases: Helimagnet (A), CSL (B), and a tilted-CSL $(\mathbf{C})$; these magnetic states are shown in Fig. 5(a). Using these configurations, we evaluate the topological charge density between nearest neighbors:

$$
\mathbf{Q}_{i}=\vec{m}_{i-1} \cdot\left(\vec{m}_{i} \times \vec{m}_{i+1}\right) .
$$

The results are shown in Fig. 5(b), where we found that $\mathbf{Q}_{i}$ systematically vanishes for the configurations (A) and (B), while a finite contribution is present for $(\mathbf{C})$. Thus, as a consequence of applying a magnetic field in the $(x z)$ plane the magnetic state becomes noncoplanar. Furthermore, in these metallic systems, coupling beyond nearest neighbors is also possible, thus more contributions to the THE are present. To summarize, both the PHE and THE are consequences of the tilted CSL. Furthermore, a vector chirality contribution $\left(\vec{m}_{i} \times \vec{m}_{j}\right)$ to the Hall effect can be relevant in the noncentrosymmetric systems treated in this paper; this was discussed in Ref. [48]. However, for the sake of simplicity, we only interpret the experimental data in terms of the THE. 
[1] V. P. Ningrum, B. Liu, W. Wang, Y. Yin, Y. Cao, C. Zha, H. Xie, X. H. Jiang, Y. Sun, S. Qin, X. Chen, T. Qin, C. Zhu, L. Wang, and W. Huang, Recent advances in two-dimensional magnets: Physics and devices towards spintronic applications, Research 2020, 1768918 (2020).

[2] K. F. Mak, J. Shan, and D. C. Ralph, Probing and controlling magnetic states in 2D layered magnetic materials, Nat. Rev. Phys. 1, 646 (2019).

[3] S. Mühlbauer, B. Binz, F. Jonietz, C. Pfleiderer, A. Rosch, A. Neubauer, R. Georgii, and P. Böni, Skyrmion lattice in a chiral magnet, Science 323, 915 (2009).

[4] K. Karube, J. S. White, N. Reynolds, J. L. Gavilano, H. Oike, A. Kikkawa, F. Kagawa, Y. Tokunaga, H. M. Ronnow, Y. Tokura, and Y. Taguchi, Robust metastable skyrmions and their triangular-square lattice structural transition in a high-temperature chiral magnet, Nat. Mater. 15, 1237 (2016)

[5] I. Kézsmárki, S. Bordács, P. Milde, E. Neuber, L. M. Eng, J. S. White, H. M. Rønnow, C. D. Dewhurst, M. Mochizuki, K. Yanai, H. Nakamura, D. Ehlers, V. Tsurkan, and A. Loidl, Néeltype skyrmion lattice with confined orientation in the polar magnetic semiconductor $\mathrm{GaV}_{4} \mathrm{~S}_{8}$, Nat. Mater. 14, 1116 (2015).

[6] R. Saha, A. K. Srivastava, T. Ma, J. Jena, P. Werner, V. Kumar, C. Felser, and S. S. P. Parkin, Intrinsic stability of magnetic anti-skyrmions in the tetragonal inverse Heusler compound $\mathrm{Mn}_{1.4} \mathrm{Pt}_{0.9} \mathrm{Pd}_{0.1} \mathrm{Sn}$, Nat. Commun. 10, 5305 (2019).

[7] T. Lui, V. Puliafito, F. Montaigne, S. Petit, C. Deranlot, S. Andrieu, O. Ozatay, G. Finocchio, and T. Hauet, Reproducible formation of single magnetic bubbles in an array of patterned dots, J. Phys D: Appl. Phys. 49, 245002 (2016).

[8] V. S. Pribaig, I. N. Krivorotov, G. D. Fuchs, P. M. Braganca, O. Ozatay, J. C. Sankey, D. C. Ralph, and R. A. Buhrman, Magnetic vortex oscillator driven by d.c. spin-polarized current, Nat. Phys. 3, 498 (2007).

[9] I. Dzyaloshinsky, A thermodynamic theory of "weak" ferromagnetism of antiferromagnetics, J. Phys. Chem. Solids 4, 241 (1958).

[10] T. Moriya, Anisotropic superexchange interaction and weak ferromagnetism, Phys. Rev. 120, 91 (1960).

[11] S. Heinze, K. von Bergmann, M. Menzel, J. Brede, A. Kubetzka, R. Wiesendanger, G. Bihlmayer, and S. Blügel, Spontaneous atomic-scale magnetic skyrmion lattice in two dimensions, Nat. Phys. 7, 713 (2011).

[12] R. Ozawa, S. Hayami, and Y. Motome, Zero-Field Skyrmions with a High Topological Number in Itinerant Magnets, Phys. Rev. Lett. 118, 147205 (2017).

[13] S. Grytsiuk, J.-P. Hanke, M. Hoffmann, J. Bouaziz, O. Gomonay, G. Bihlmayer, S. Lounis, Y. Mokrousov, and S. Blügel, Topological-chiral magnetic interactions driven by emergent orbital magnetism, Nat. Commun. 11, 511 (2020).

[14] T. Okubo, S. Chung, and H. Kawamura, Multiple- $q$ States and the Skyrmion Lattice of the Triangular-Lattice Heisenberg Antiferromagnet Under Magnetic Fields, Phys. Rev. Lett. 108, 017206 (2012).

[15] A. O. Leonov and M. Mostovoy, Multiply periodic states and isolated skyrmions in an anisotropic frustrated magnet, Nat. Commun. 6, 8275 (2015).

[16] N. Kanazawa, Y. Onose, T. Arima, D. Okuyama, K. Ohoyama, S. Wakimoto, K. Kakurai, S. Ishiwata, and Y. Tokura, Large
Topological Hall Effect in a Short-Period Helimagnet MnGe, Phys. Rev. Lett. 106, 156603 (2011).

[17] K. Nakazawa, M. Bibes, and H. Kohno, Topological Hall effect from strong to weak coupling, J. Phys. Soc. Jpn. 87, 033705 (2018).

[18] Y. Taguchi, Y. Oohara, H. Yoshizawa, N. Nagaosa, and Y. Tokura, Spin chirality, Berry phase, and anomalous Hall effect in a frustrated ferromagnet, Science 291, 2573 (2001).

[19] C. S. Spencer, J. Gayles, N. A. Porter, S. Sugimoto, Z. Aslam, C. J. Kinane, T. R. Charlton, F. Freimuth, S. Chadov, S. Langridge, J. Sinova, C. Felser, S. Blügel, Y. Mokrousov, and C. H. Marrows, Helical magnetic structure and the anomalous and topological Hall effects in epitaxial B20 $\mathrm{Fe}_{(1-\mathrm{y})} \mathrm{Co}_{\mathrm{y}} \mathrm{Ge}$ films, Phys. Rev. B 97, 214406 (2018).

[20] A. Neubauer, C. Pfleiderer, B. Binz, A. Rosch, R. Ritz, P. G. Niklowitz, and P. Böni, Topological Hall Effect in the $A$ Phase of MnSi, Phys. Rev. Lett. 102, 186602 (2009).

[21] N. A. Porter, J. C. Gartside, and C. H. Marrows, Scattering mechanisms in textured FeGe thin films: Magnetoresistance and the anomalous Hall effect, Phys. Rev. B 90, 024403 (2014).

[22] T. Kurumaji, T. Nakajima, M. Hirschberger, A. Kikkawa, Y. Yamasaki, H. Sagayama, H. Nakao, Y. Taguchi, T. Arima, and Y. Tokura, Skyrmion lattice with a giant topological Hall effect in a frustrated triangular-lattice magnet, Science 365, 914 (2019).

[23] T. Schulz, R. Ritz, A. Bauer, M. Halder, M. Wagner, C. Franz, C. Pfleiderer, K. Everschor, M. Garst, and A. Rosch, Emergent electrodynamics of skyrmions in a chiral magnet, Nat. Phys. 8, 301 (2012).

[24] S. Wang, Q. Zeng, D. Liu, H. Zhang, L. Ma, G. Xu, Y. Liang, Z. Zhang, H. Wu, R. Che, X. Han, and Q. Huang, Giant topological Hall effect and superstable spontaneous skyrmions below $330 \mathrm{~K}$ in a centrosymmetric complex noncollinear ferromagnet $\mathrm{NdMn}_{2} \mathrm{Ge}_{2}$, ACS Appl. Mater. Interfaces 12, 24125 (2020).

[25] L. Vistoli, W. Wang, A. Sander, Q. Zhu, B. Casals, R. Cichelero, A. Barthélémy, S. Fusil, G. Herranz, S. Valencia et al., Giant topological Hall effect in correlated oxide thin films, Nat. Phys. 15, 67 (2019).

[26] Y. Togawa, T. Koyama, K. Takayanagi, S. Mori, Y. Kousaka, J. Akimitsu, S. Nishihara, K. Inoue, A. S. Ovchinnikov, and J. Kishine, Chiral Magnetic Soliton Lattice on a Chiral Helimagnet, Phys. Rev. Lett. 108, 107202 (2012).

[27] S. S. P. Parkin and R. H. Friend, 3d transition-metal intercalates of the niobium and tantalum dichalcogenides. I. Magnetic properties, Philos. Mag. B 41, 65 (1980).

[28] S. S. P. Parkin and R. H. Friend, 3d transition-metal intercalates of the niobium and tantalum dichalcogenides. II. Transport properties, Philos. Mag. B 41, 95 (1980).

[29] Y. Cao, Z. Huang, Y. Yin, H. Xie, B. Liu, B. Wang, C. Zhu, D. Mandrus, L. Wang, and W. Huang, Overview and advances in a layered chircal helimagnet $\mathrm{Cr}_{1 / 3} \mathrm{NbS}_{2}$, Mater. Today Adv. 7, 100080 (2020).

[30] Y. Togawa, T. Koyama, Y. Nishimori, Y. Matsumoto, S. McVitie, D. McGrouther, R. L. Stamps, Y. Kousaka, J. Akimitsu, S. Nishihara, K. Inoue, I. G. Bostrem, Vl. E. Sinitsyn, A. S. Ovchinnikov, and J. Kishine, Magnetic soliton confinement and discretization effects arising from macroscopic coherence in a chiral spin soliton lattice, Phys. Rev. B 92 220412(R) (2015). 
[31] J.-i. Yonemura, Y. Shimamoto, T. Kida, D. Yoshizawa, Y. Kousaka, S. Nishihara, F. J. T. Goncalves, J. Akimitsu, K. Inoue, M. Hagiwara, and Y. Togawa, Magnetic solitons and magnetic phase diagram of the hexagonal chiral crystal $\mathrm{CrNb}_{3} \mathrm{~S}_{6}$ in oblique magnetic fields, Phys. Rev. B 96, 184423 (2017).

[32] See Supplemental Material at http://link.aps.org/supplemental/ 10.1103/PhysRevResearch.4.013134 for methodological details and supporting experimental results and theoretical simulations.

[33] R. V. Colvin, S. Legvold, and F. H. Spedding, Electrical resistivity of the heavy rare-earth metals, Phys. Rev. 120, 741 (1960).

[34] M. Barati, W. R. Datars, T. R. Chien, C. V. Stager, and J. D. Garrett, Resistivity, Hall effect, and magnetic susceptibility of $\mathrm{UPd}_{2} \mathrm{Si}_{2}$, Phys. Rev. B 48, 16926 (1993).

[35] C. Haas, Spin-disorder scattering and magnetoresistance of magnetic semiconductors, Phys. Rev. 168, 531 (1968).

[36] N. J. Ghimire, M. A. McGuire, D. S. Parker, B. Sipos, S. Tang, J.-Q. Yan, B. C. Sales, and D. Mandrus, Magnetic phase transition in single crystals of the chiral helimagnet $\mathrm{Cr}_{1 / 3} \mathrm{NbS}_{2}$, Phys. Rev. B 87, 104403 (2013).

[37] V. Dyadkin, F. Mushenok, A. Bosak, D. Menzel, S. Grigoriev, P. Pattison, and D. Chernyshov, Structural disorder versus chiral magnetism in $\mathrm{Cr}_{1 / 3} \mathrm{NbS}_{2}$, Phys. Rev. B 91, 184205 (2015).

[38] M. Lee, Y. Onose, Y. Tokura, and N. P. Ong, Hidden constant in the anomalous Hall effect of high-purity magnet MnSi, Phys. Rev. B 75, 172403 (2007).

[39] B. J. Chapman, M. G. Grossnickle, T. Wolf, and M. Lee, Large enhancement of emergent magnetic fields in $\mathrm{MnSi}$ with impurities and pressure, Phys. Rev. B 88, 214406 (2013).

[40] H. Zhang, Q. Huang, L. Hao, J. Yang, K. Noordhoek, S. Pandey, H. Zhou, and J. Liu, Anomalous magnetoresistance in centrosymmetric skyrmion-lattice magnet $\mathrm{Gd}_{2} \mathrm{PdSi}_{3}$, New J. Phys. 22, 083056 (2020).

[41] G. P. Müller, M. Hoffmann, C. Dißelkamp, D. Schürhoff, S. Mavros, M. Sallermann, N. S. Kiselev, H. Jónsson, and S. Blügel, SPIRIT: Multifunctional framework for atomistic spin simulations, Phys. Rev. B 99, 224414 (2019).

[42] M.-C. Chang and Q. Niu, Berry phase, hyperorbits, and the Hofstadter spectrum: Semiclassical dynamics in magnetic Bloch bands, Phys. Rev. B. 53, 7010 (1996).

[43] N. Nagaosa, J. Sinova, S. Onoda, A. H. MacDonald, and N. P. Ong, Anomalous Hall effect, Rev. Mod. Phys. 82, 1539 (2010).

[44] J. Smit, The spontaneous Hall effect in ferromagnetics I, Physica 21, 877 (1955).

[45] L. Berger, Side-jump mechanism for the Hall effect of ferromagnets, Phys. Rev. B 2, 4559 (1970).

[46] B. Ernst, R. Sahoo, Y. Sun, J. Nayak, L. Müchler, A. K. Nayak, N. Kumar, J. Gayles, A. Markou, G. H. Fecher, and C. Felser, Anomalous Hall effect and the role of Berry curvature in $\mathrm{Co}_{2}$ TiSn Heusler films, Phys. Rev. B 100, 054445 (2019).

[47] P. Bruno, V. K. Dugaev, and M. Taillefumier, Topological Hall Effect and Berry Phase in Magnetic Nanostructures, Phys. Rev. Lett. 93, 096806 (2004).
[48] J. Bouaziz, H. Ishida, S. Lounis, and S. Blügel, Transverse Transport in Two-Dimensional Relativistic Systems with Nontrivial Spin Textures, Phys. Rev. Lett. 126, 147203 (2021).

[49] J.-P. Jan, Galvamomagnetic and thermomagnetic effects in metals, in Solid State Physics (Elsevier, New York, 1957), Vol. 5, pp. 1-96.

[50] K. M. Seemann, F. Freimuth, H. Zhang, S. Blügel, Y. Mokrousov, D. E. Bürgler, and C. M. Schneider, Origin of the Planar Hall Effect in Nanocrystalline $\mathrm{Co}_{60} \mathrm{Fe}_{20} \mathrm{~B}_{20}$, Phys. Rev. Lett. 107, 086603 (2011).

[51] J. Bouaziz, S. Lounis, S. Blügel, and H. Ishida, Microscopic theory of the residual surface resistivity of Rashba electrons, Phys. Rev. B 94, 045433 (2016).

[52] Z. Li, T. Xiao, R. Zou, J. Li, Y. Zhang, Y. Zeng, M. Zhou, J. Zhang, and W. Wu, Planar Hall effect in $\mathrm{PtSe}_{2}$, J. Appl. Phys. 127, 054306 (2020).

[53] Y. Togawa, Y. Kousaka, S. Nishihara, K. Inoue, J. Akimitsu, A. S. Ovchinnikov, and J. Kishine, Interlayer Magnetoresistance Due to Chiral Soliton Lattice Formation in Hexagonal Chiral Magnet $\mathrm{CrNb}_{3} \mathrm{~S}_{6}$, Phys. Rev. Lett. 111, 197204 (2013).

[54] H. Han, L. Zhang, D. Sapkota, N. Hao, L. Ling, H. Du, L. Pi, C. Zhang, D. G. Mandrus, and Y. Zhang, Tricritical point and phase diagram based on critical scaling in the monoaxial chiral helimagnet $\mathrm{Cr}_{1 / 3} \mathrm{NbS}_{2}$, Phys. Rev. B 96, 094439 (2017).

[55] A. C. Bornstein, B. J. Chapman, N. J. Ghimire, D. G. Mandrus, D. S. Parker, and M. Lee, Out-of-plane spin-orientation dependent magnetotransport properties in the anisotropic helimagnet $\mathrm{Cr}_{1 / 3} \mathrm{NbS}_{2}$, Phys. Rev. B 91, 184401 (2015).

[56] D. Maryenko, A. S. Mishchenko, M. S. Bahramy, A. Ernst, J. Falson, Y. Kozuka, A. Tsukazaki, N. Nagaosa, and M. Kawasaki, Observation of anomalous Hall effect in nonmagnetic two-dimensional electron system, Nat. Commun. 8, 14777 (2017).

[57] S. Onoda, N. Sugimoto, and N. Nagaosa, Quantum transport theory of anomalous electric, thermoelectric, and thermal Hall effects in ferromagnets, Phys. Rev. B 77, 165103 (2008).

[58] H. Ishizuka and N. Nagaosa, Spin chirality induced skew scattering and anomalous Hall effect in chiral magnets, Sci. Adv. 4, eaap9962 (2018)

[59] Y. Li, N. Kanazawa, X. Z. Yu, A. Tsukazaki, M. Kawasaki, M. Ichikawa, X. F. Jin, F. Kagawa, and Y. Tokura, Robust Formation of Skyrmions and Topological Hall Effect Anomaly in Epitaxial Thin Films of MnSi, Phys. Rev. Lett. 110, 117202 (2013).

[60] G. W. Paterson, A. A. Tereshchenko, S. Nakayama, Y. Kousaka, J. Kishine, S. McVitie, A. S. Ovchinnikov, I. Proskurin, and Y. Togawa, Tensile deformations of the magnetic chiral soliton lattice probed by Lorentz transmission electron microscopy, Phys. Rev. B 101, 184424 (2020).

[61] K. Tsuruta, M. Mito, H. Deguchi, J. Kishine, Y. Kousaka, J. Akimitsu, and $\mathrm{K}$. Inoue, Phase diagram of the chiral magnet $\mathrm{Cr}_{1 / 3} \mathrm{NbS}_{2}$ in a magnetic field, Phys. Rev. B 93, 104402 (2016).

[62] See http://wrap.warwick.ac.uk/162181/. 